\title{
Look-alike galaxies:
}

\section{HI observations for look-alike galaxies of four calibrators ${ }^{\star}$}

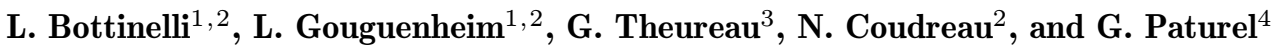 \\ 1 Université Paris-Sud, F-91405 Orsay, France \\ 2 Observatoire de Paris-Meudon, URA 1757, F-92195 Meudon Principal Cedex, France \\ 3 Osservatorio di Capodimonte, via Moiariello 16, I-80131 Napoli, Italy \\ 4 CRAL-Observatoire de Lyon, UMR 5574, F-69230 Saint-Genis Laval, France
}

Received August 17; accepted November 16, 1998

\begin{abstract}
We present a programme aiming at applying the Tully-Fisher relation for galaxies with the same morphological type and the same inclination (look-alike galaxies or sosie galaxies) as calibrating galaxies. The advantage of sosie galaxies is discussed. In particular, it is shown that using sosies of bright calibrators will allow us to explore the universe deeper and more efficiently than the classical TF method applied to different morphological types and different inclinations. As a preliminary part, we report in this paper new HI observations performed with the radiotelescope of Nançay (France) for sosies of four calibrators NGC 224, NGC 3031, NGC 253 and NGC 5457. 82 galaxies were detected.
\end{abstract}

Key words: galaxies: general — radio lines: galaxies astronomical data bases: miscellaneous - cosmology: distance scale

\section{Introduction}

Most of the methods used for extragalactic distance determination are based on a linear relationship between the absolute magnitude $M$ and a given observable parameter $P$.

$M=a \log P+b$.

Send offprint requests to: G. Paturel

* These observations made use of Nançay radiotelescope. The Nançay Radio Observatory is the Unité scientifique de Nançay of the Observatoire de Paris,associated as Unité de Service et de Recherche (USR) No. B704 to the French Centre National de la Recherche Scientifique (CNRS). The Nançay Observatory also acknowledges the financial support of the Conseil Régional of the Région Centre in France.
The distance modulus is thus derived from the classical relation

$\mu=m^{\mathrm{c}}-M$

where $m^{\mathrm{c}}$ is the apparent magnitude corrected for perturbing effects (inclination, redshift, galactic extinction...) Similarly, the parameter $P$ must also be corrected for such effects. Without considering the galactic extinction, these corrections depend essentially on axis ratio and morphological type. For spiral galaxies, one of the best parameters $P$ is the 21-cm line width (Tully \& Fisher 1977).

The principle of the method of sosie galaxies ${ }^{1}$ (Paturel 1981; Sandage 1996) consists in selecting galaxies having similar observational properties as calibrating galaxies. For instance, one can select galaxies having the same morphological type, the same axis ratio and the same $21-\mathrm{cm}$ line width, as a given calibrating galaxy (e.g., M 31). From Rel. 1, these galaxies have the same absolute magnitude as the considered calibrating galaxy. This method has revealed to be a powerful tool in obtaining accurate galaxy distances because it does not assume anything about the expression of, e.g. the Tully-Fisher relationship. For instance, the value of $a$ or $b$ does not intervene in the final result. Any morphological type dependences are removed etc.

We started a new observational program to search for new sosie candidates. This means that we are observing galaxies with at least the same morphological type and the same axis ratio as calibrators, but, obviously not necessarily the same 21-cm line width. Among detected galaxies, some will be pure sosies of the calibrators (i.e. galaxies with the same $21-\mathrm{cm}$ line width), others not. This last class will be called "sosie candidates". Nevertheless, sosie candidates can also be used through the conventional TF relation with the still valid advantage that the result does not depend on morphological type and inclination effects.

\footnotetext{
1 Look-alike in English.
} 




Fig. 1. Completeness curve for all sosie candidates of M 31 (filled circles) extracted from LEDA database. The completeness is fullfilled up to $\approx 15 \mathrm{Bmag}$ (vertical line on the right). Today, only a part of this sample is observed in HI (open circles) and the completeness of this subsample is fullfilled only up to an apparent magnitude of about $12.5 \mathrm{Bmag}$. (vertical line on the left). The dashed line represents the idealized completeness curve

Only, uncertainty about the value of the slope $a$ may affect the final result which anyway will be more secure.

Another problem still plagues the use of any distance determination. It has been shown that Malmquist bias appears at very small distances. For instance, for galaxies as luminous as M 31, the bias starts for radial velocities as small as $2000 \mathrm{~km} \mathrm{~s}^{-1}$. To push this limit deeper we will enlarge our sample and observe galaxies up to fainter apparent magnitude.

In order to check the effect of the bias it is compulsory to work with a sample complete up to a well determined apparent magnitude. We present in Fig. 1 the completeness curve for all sosie candidates of M 31 extracted from the LEDA database. This sample contains 1434 galaxies. The completeness curve (filled circles) is drawn. On the same figure the completeness curve is given for those 351 galaxies of the sample having known $21-\mathrm{cm}$ line width (open circles). This shows that more than 1083 galaxies have still to be observed to get all sosie candidates of M 31 (hence, all sosies of M 31) up to an apparent $B$-magnitude 15. Fortunately, among these 1083 galaxies, 367 already have an optical radial velocity and will be easy to observe in HI without having to use the search mode. They will constitute our first priority target.

In order to show the benefit of using a deeper sample, we plot in Fig. 2 the apparent Hubble constant $H_{0}$ vs. the radial velocity for M 31 sosie galaxies and four different completeness limits $\left(B_{\lim }=12,13,14\right.$ and 15). These curves show the effect of the Malmquist bias under different conditions. They are calculated according to Teerikorpi (1975, Eq. (5)) using a dispersion of the Tully-Fisher relation of $\sigma=0.6$ and a Hubble constant of

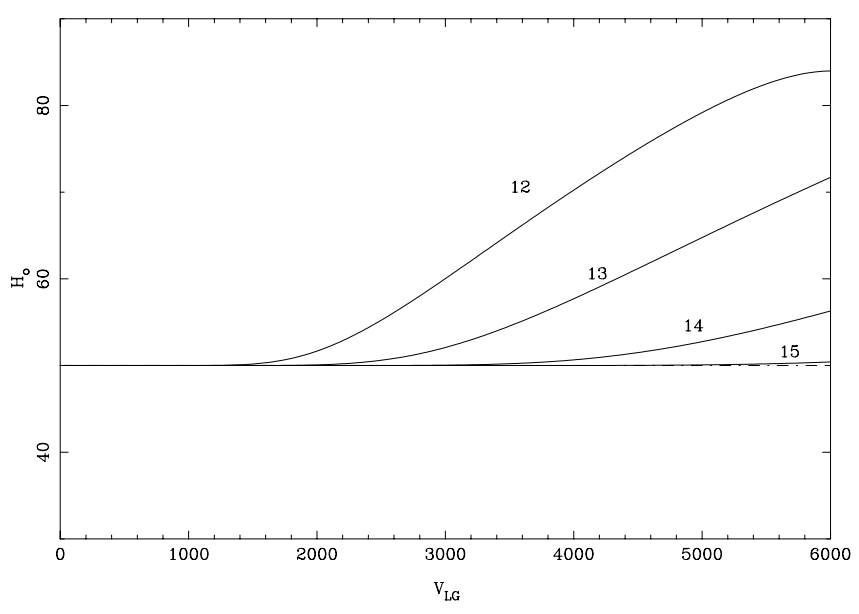

Fig. 2. Bias curves of $M$ 31-sosies for four completeness limits: $B_{\lim }=12,13,14$ and 15 . It appears that the bias is negligibly small up to a velocity of $6000 \mathrm{~km} \mathrm{~s}^{-1}$ when the sample is complete up to a limit of $B_{\text {lim }}=15$

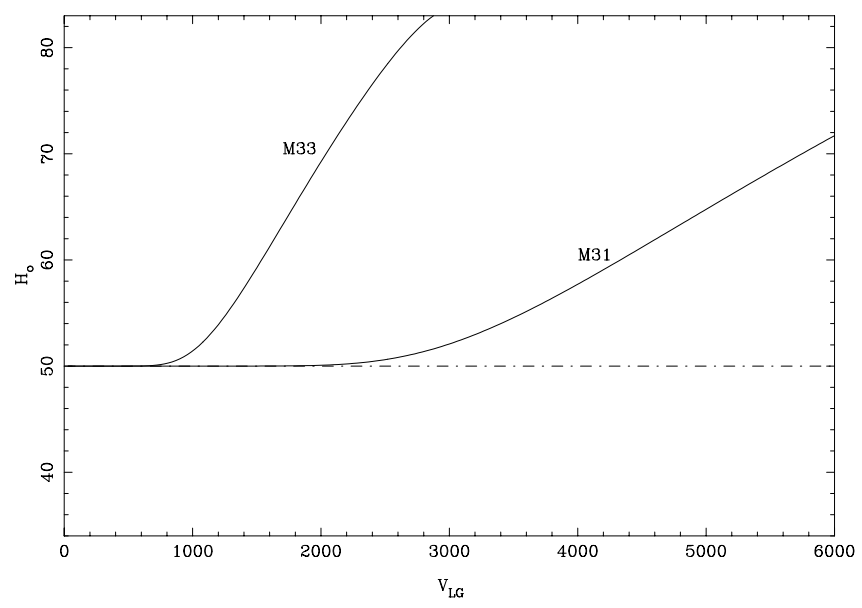

Fig. 3. Bias curves of M 31-sosies and M 33-sosies for a completeness limits $B_{\lim }=13$. It appears that the bias is much stronger for M 33 which is less luminous than M 31

$50 \mathrm{~km} \mathrm{~s}^{-1} \mathrm{Mpc}^{-1}$. It appears clearly that the bias would be negligible up to a radial velocity of $6000 \mathrm{~km} \mathrm{~s}^{-1}$ if a completeness limit of $B_{\lim }=15$ is reached. The Hubble constant derived in this way would not be influenced very much by large scale flows, because these flows are generally assumed to be smaller than about $600 \mathrm{~km} \mathrm{~s}^{-1}$. Note that, the bias effect would be much stronger if sosies of a less luminous calibrator were used.

For instance, we compare the bias for M 33 and M 31 (Fig. 3) using the same bias curve as above but a completeness limit of $B_{\lim }=13$. It is obvious that it is better to use a luminous calibrator.

A preliminary study from pure sosies of M 31 and M 81 is presented in a companion paper (Paturel et al. 1998) where distances to the calibrators come from Cepheid Period-Luminosity relation calibrated with geometrical parallaxes. Another study is in preparation with all available sosie candidates. This program will be pursued by 


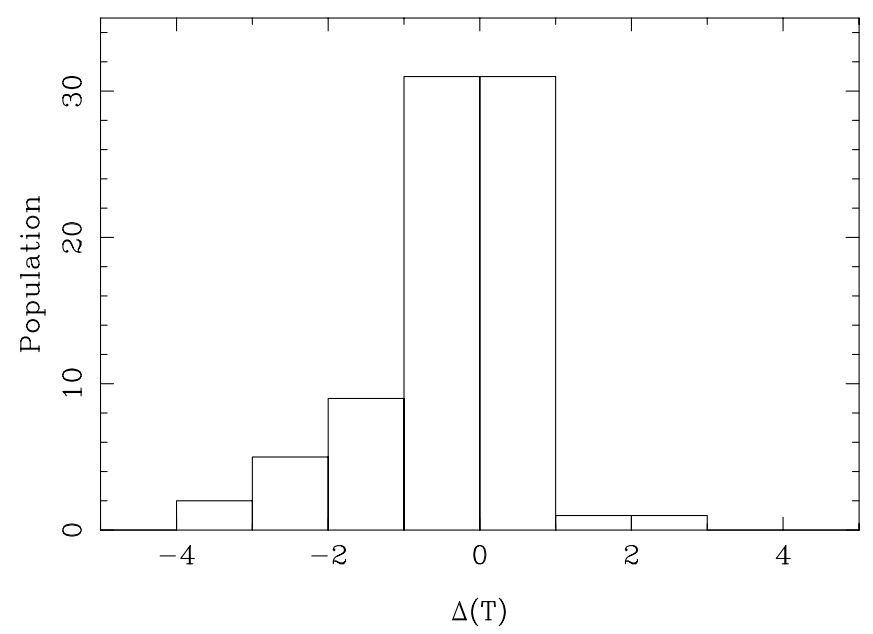

Fig. 4. Histogram of deviations in morphological types $\Delta T=$ $T$ (look-alike $)-T$ (calibrator). The standard deviation is \pm 1.0

systematic HI observations of sosie candidates of different calibrators. In this paper we present a first step of observations obtained for this program. We started it by searching sosie candidates of two intrinsically luminous galaxies (M 31 and M 81), one low luminosity galaxy (NGC 253) and one face-on galaxy (NGC 5457).

The look-alike galaxies are far more distant than the calibrating galaxies. Hence morphological classification and axis ratio measurements are less certain. Further, the morphological types and axis ratios of galaxies of our program are revised from time to time due to inclusion of new data. The morphological type is coded numerically with $T$ according to de Vaucouleurs et al. (1976). The axis ratio is noted $R_{25}$ and corresponds to the ratio of the major to the minor axis of the external isophote at the limiting surface brightness of $25 \mathrm{Bmag} \operatorname{arcsec}^{-2}$. In Figs. 4 and 5 we present the histograms of $\Delta T=T$ (lookalike) $-T$ (calibrator) and $\Delta \log R_{25}=\log R_{25}$ (look alike) $-\log R_{25}$ (calibrator) in order to show the actual uncertainty in the definition of a look-alike candidate. The standart deviations of these quantities, $\sigma(\Delta T)=1.0$ and $\sigma\left(\Delta \log R_{25}\right)=0.07$, correspond to the typical error of $T$ and $\log R_{25}$, respectively but two galaxies are rejected afterwards (PGC 06289 and PGC 68360).

\section{HI observations}

\subsection{HI velocity measurements}

The technical procedure has been described in Fouqué et al. (1990). The main characteristics of the Nançay radiotelescope receiver are given in Table 1.

The Nançay radiotelescope has a non circular HalfPower-Beam-Width (HPBW) of roughly $\leq 22^{\prime} \mathrm{NS} \times 4^{\prime} \mathrm{EW}$ which actually depends on the elevation along the NorthSouth direction. The variation is presented in Fig. 6 (E. Gérard, private communication). The change of the

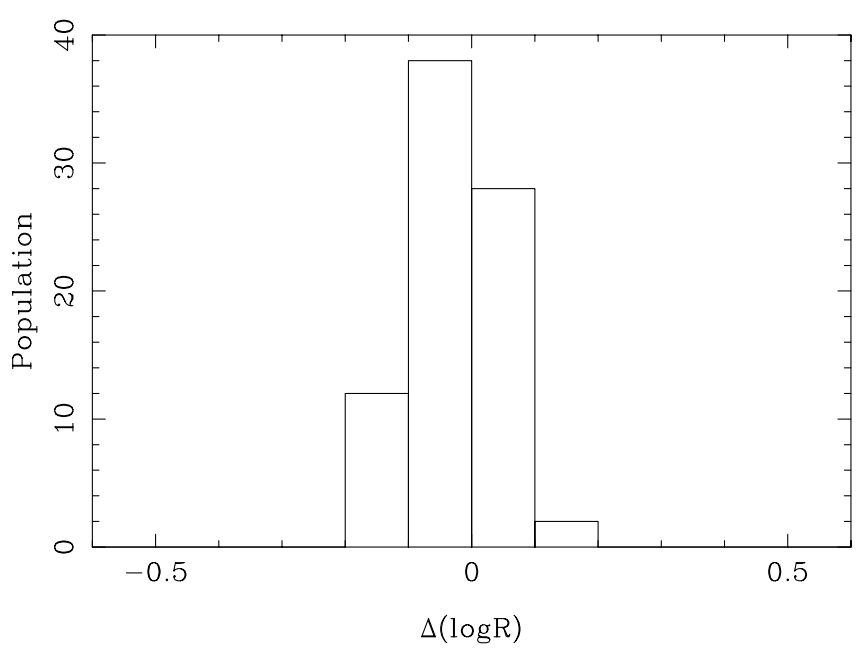

Fig. 5. Histogram of deviations in axis ratios $\Delta \log R_{25}=$ $\log R_{25}$ (look - alike) - $\log R_{25}$ (calibrator). The standard deviation is \pm 0.07

Table 1. Characteristics of the Nançay radiotelescope receiver

\begin{tabular}{ll}
\hline HPBW at 21-cm (see text) & $\approx 3.6^{\prime} \times 22.0^{\prime}$ \\
minimum temperature & $37 \mathrm{~K}$ \\
Total number of channels & 1024 \\
Number of channels (search mode) & $4 \times 256$ \\
Number of channels (measure) & $2 \times 512(2$ polar.) \\
Total passband & $6.4 \mathrm{MHz}$ \\
Spacing of channels (at 21-cm) & $2.6 \mathrm{~km} \mathrm{~s}^{-1}$ \\
Final resolution (after smoothing) & $10 \mathrm{~km} \mathrm{~s}^{-1}$ \\
\hline
\end{tabular}

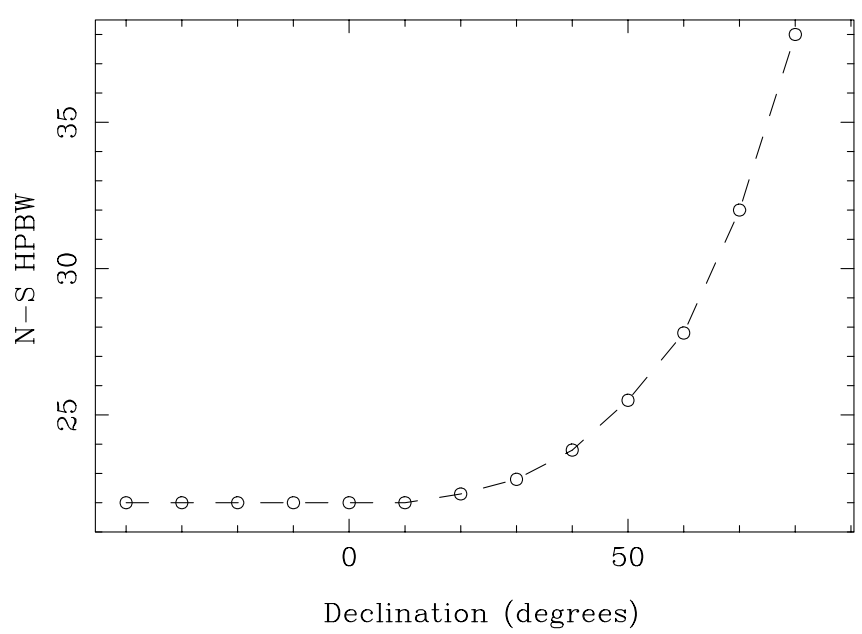

Fig. 6. Variation of the North-South Half-Power-Beam-Width of the Nançay radiotelescope according to E. Gérard (private communication)

HPWB intervenes for estimating the confusion of a measurement and for the beam filling correction. In this paper, only raw fluxes are given (i.e. uncorrected for beam-filling effect). It is worth noting that, the beam-filling correction also depends on the position angle of the galaxy as described in Bottinelli et al. (1990). 
Annexe: 21-cm line profiles for sosie candidates of NGC 224 (Fig. 7), NGC 3031 (Fig. 8), NGC 253 (Fig. 10) and NGC 5457 (Fig. 9). The $x$-axis gives the heliocentric radial velocity $c \Delta \lambda / \lambda$ in $\mathrm{km} \mathrm{s}^{-1}$. The $y$-axis gives the flux density in mJy
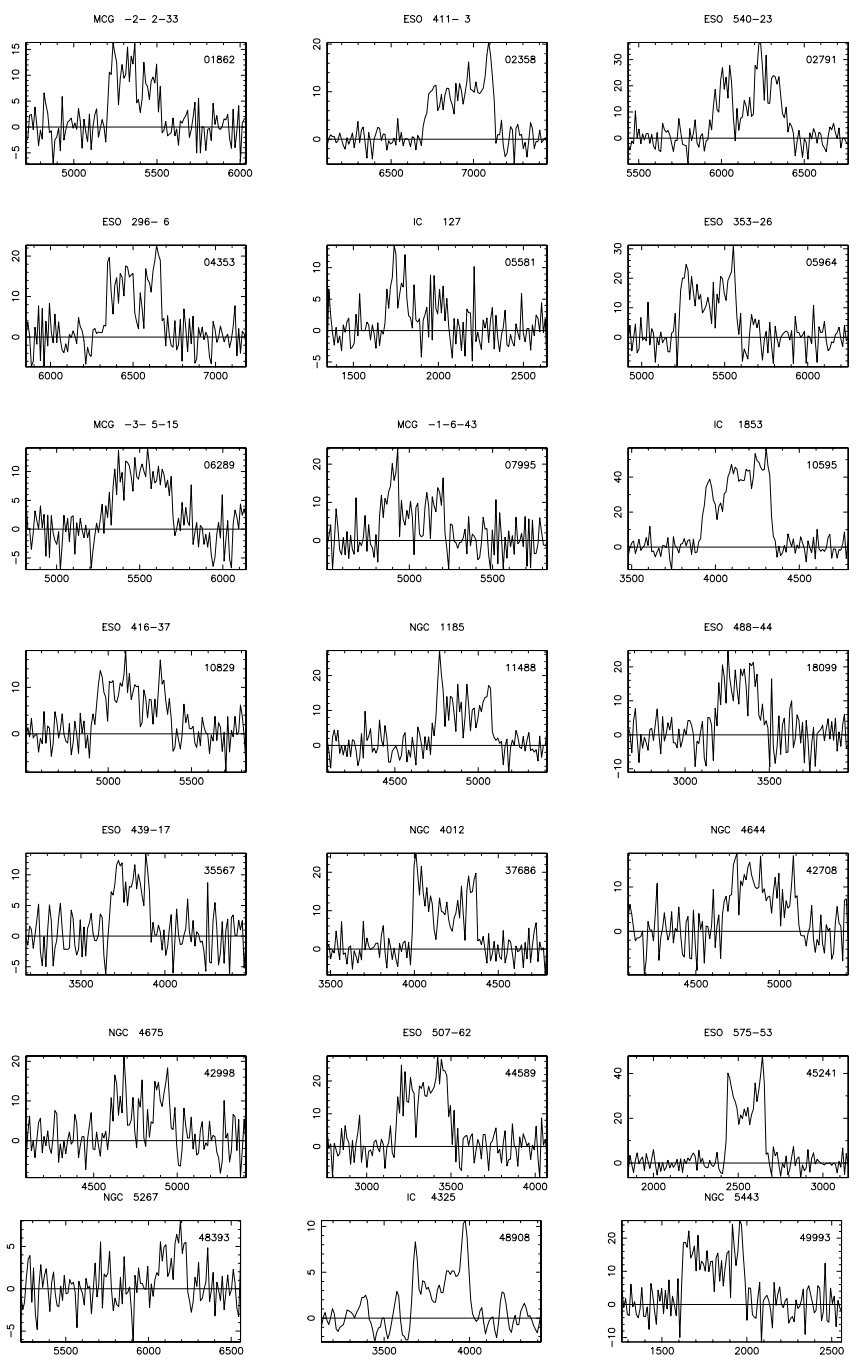

ESO $446-4$
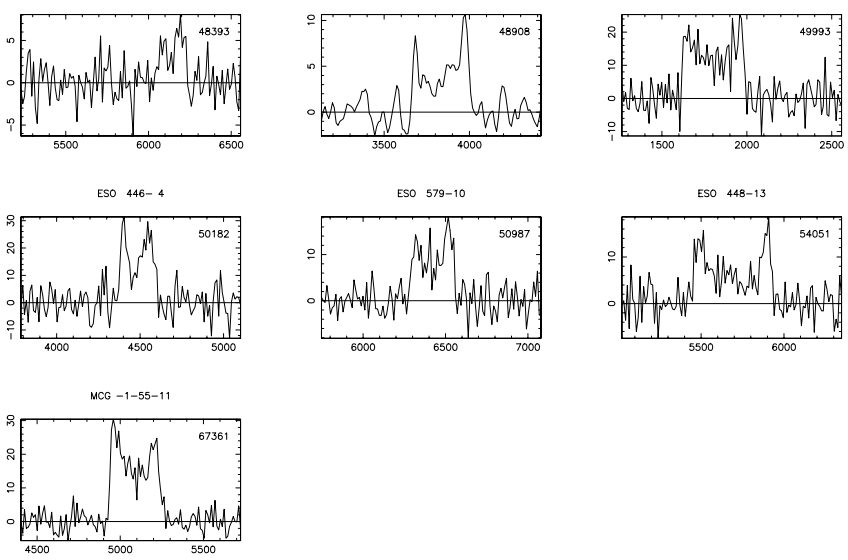

Fig. 7. 21-cm line profiles for sosie candidates of NGC 224
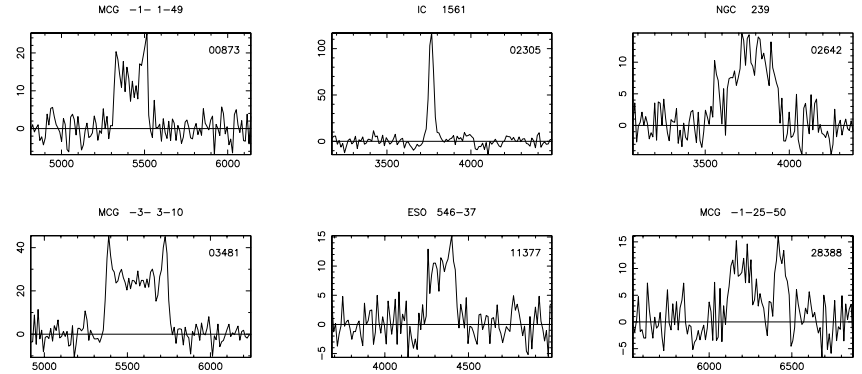

ESO $501-12$
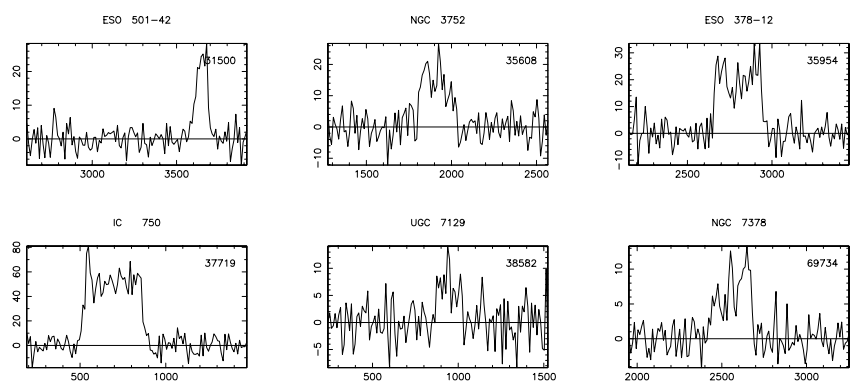

Fig. 8. 21-cm line profiles for sosie candidates of NGC 3031
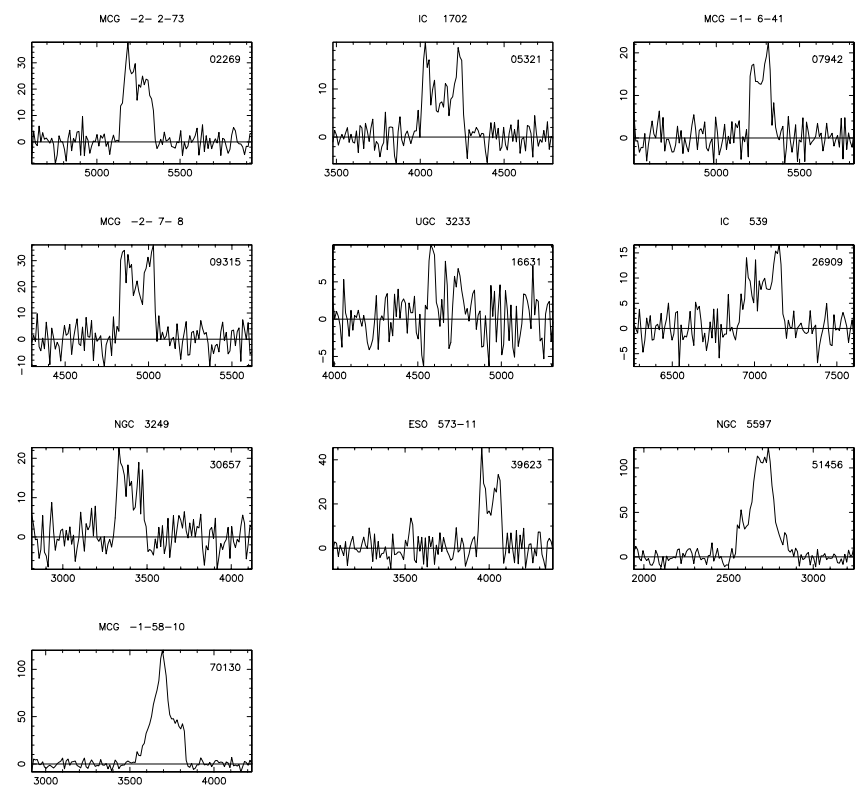

Fig. 9. 21-cm line profiles for sosie candidates of NGC 5457

The 21-cm line widths are measured using an interactive method. The smoothed 21-cm line is displayed on a screen; the user estimates visually the maximum of the profile; the program calculates the width at $20 \%$ and $50 \%$ of this maximum by considering the points where both levels intercept the profile. This procedure is impossible when the $\mathrm{S} / \mathrm{N}$ ratio is poor (less than $\approx 3$ like for NGC 4675, NGC 5267 and ESO 404-15). Nevertheless, the user has full control to obtain the most secure result, for instance by smoothing the profile in noisy regions. The most critical part is the proper estimation of the maximum. 

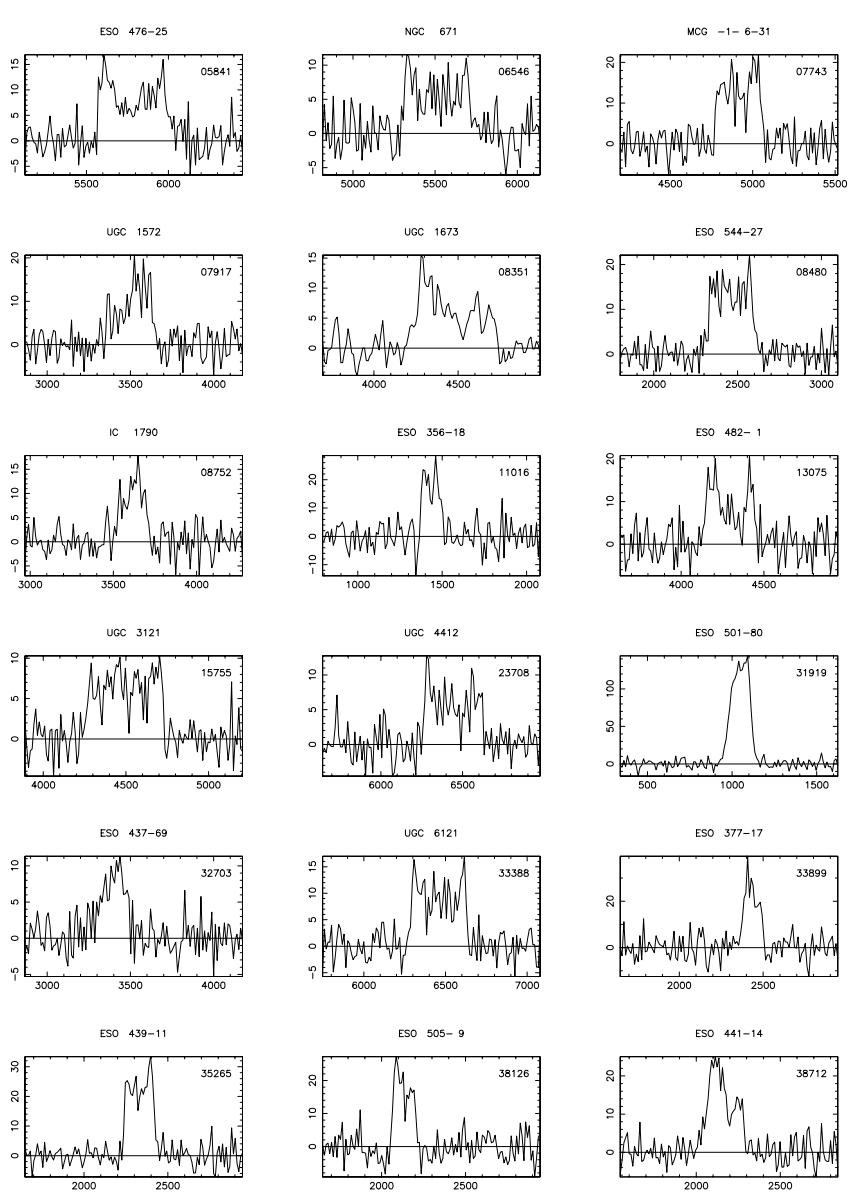

Fig. 10. 21-cm line profiles for sosie candidates of NGC 253

When the profile has two maxima at different levels, the adopted maximum is the mean of both if it is compatible with the noise (otherwise, the higher level is chosen, but it is reduced by the standard deviation of the noise).

\subsection{Results}

All new measurements are presented in Table 2. Mean errors and $\mathrm{S} / \mathrm{N}$ ratio are calculated according to Fouqué et al. (1990). Columns are arranged as it follows:

- Column 1: Identification with the PGC name (According to Paturel et al. 1989a,b).

- Column 2: Identification with an alternative name according to the following hierarchy: NGC/IC, UGC, ESO, MCG (Paturel 1989a).

- Column 3: Measured heliocentric velocity measured at the middle of the $20 \%$ level in $\mathrm{km} \mathrm{s}^{-1}$.

- Column 4: Measured heliocentric velocity measured at the middle of the $50 \%$ level in $\mathrm{km} \mathrm{s}^{-1}$.

- Column 5: Internal mean error on both V 20 and V 50.

- Column 6: 21-cm line width W 20 at $20 \%$ of the peak value in $\mathrm{km} \mathrm{s}^{-1}$.
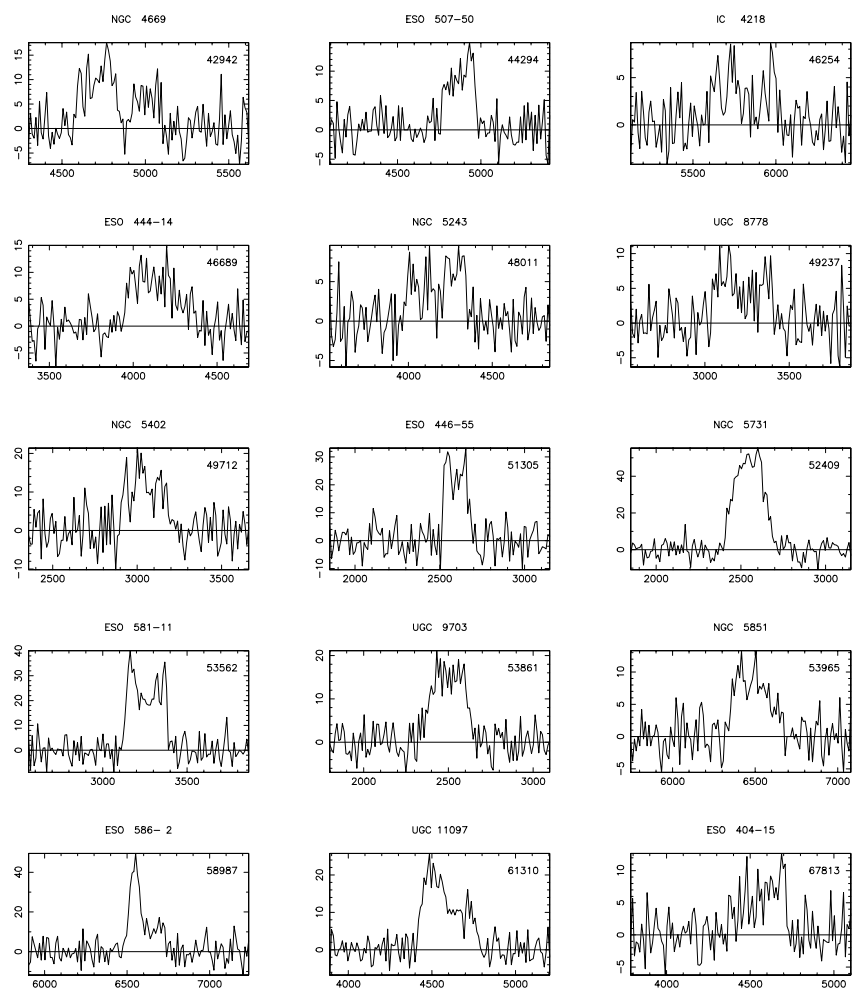

E50 $467-25$
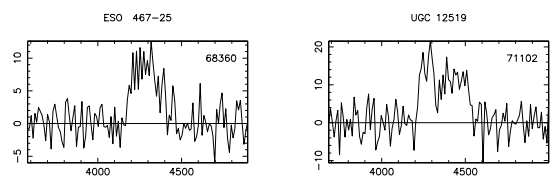

- Column \%: Internal mean error on W 20.

- Column 8: 21-cm line width W 50 at $50 \%$ of the peak value in $\mathrm{km} \mathrm{s}^{-1}$.

- Column 9: Internal mean error on W 50.

- Column 10: 21-cm flux FH (area defined by the 21-cm line profile, in $\mathrm{Jy}_{\mathrm{km} \mathrm{s}}{ }^{-1}$ ).

- Column 11: Internal mean error on FH.

- Column 12: $\mathrm{S} / \mathrm{N}$ ratio.

The HI-profiles are given in Figs. 7 to 9 of the Annexe for sosies candidates of NGC 224, NGC 3031, NGC 253 and NGC 5457, respectively. For each spectrum the galaxy name is given above the frame. The PGC name is given in the upper-right corner. The $x$-axis gives the heliocentric velocity expressed in $\mathrm{km} \mathrm{s}^{-1}$ with the optical convention $(\Delta \lambda / \lambda)$. The $y$-axis gives the flux in mJy.

\subsection{Discussion}

The confusion of HI measurements is an old problem in astronomy. For each galaxy a visual inspection of the field has been made using the charts produced with the LEDA database. The galaxies listed below are confused or possibly confused. For each of them we calculate two 
coefficients in order to quantify the degree of confusion. The first coefficient gives the degree of confusion in position. It is defined as:

$C_{\mathrm{p}}=1-\sqrt{\frac{\Delta x^{2}}{a^{2}}+\frac{\Delta y^{2}}{b^{2}}}$

where $\Delta x$ and $\Delta y$ are the angular distances (in right ascension and declination) between the observed galaxy and the galaxies of the field and where $a$ and $b$ are the HPBW in the corresponding directions. The HPBW in the $\mathrm{N}-\mathrm{S}$ direction is calculated according to Fig. 6. The second coefficient gives the degree of confusion in velocity. It is defined as:

$C_{\mathrm{v}}=1-\frac{|\Delta V|}{W_{20}}$

where $\Delta V$ is the velocity difference between the observed galaxy and the galaxies of the field and where $W_{20}$ is the 21-cm line width for the observed galaxy. A confusion exists when $C_{\mathrm{p}}$ and $C_{\mathrm{v}}$ are both larger than zero. For each confused galaxy we give the maximum value of $C_{\mathrm{p}}$ and $C_{\mathrm{v}}$.

- PGC 02791: Confused by PGC $2794=$ ESO 540-24 $\left(V=6259 \mathrm{~km} \mathrm{~s}^{-1}\right)$ and/or PGC $2796=$ ESO 540-25 $\left(V=6362 \mathrm{~km} \mathrm{~s}^{-1}\right) ; C_{\mathrm{p}}=0.83$ and $C_{\mathrm{v}}=0.96$.

- PGC 10595: Confused by PGC 10597 = NGC 1103 $\left(V=4156 \mathrm{~km} \mathrm{~s}^{-1}\right)$.

- PGC 42708: Confused by PGC $42725=$ MCG 9-21-32 $\left(V=4913 \mathrm{~km} \mathrm{~s}^{-1}\right) ; C_{\mathrm{p}}=0.79$ and $C_{\mathrm{v}}=0.97$.

- PGC 08351: Confused by PGC $08352=$ NGC $834(V=$ $\left.4594 \mathrm{~km} \mathrm{~s}^{-1}\right) ; C_{\mathrm{p}}=0.59$ and $C_{\mathrm{v}}=0.58$.

- PGC 02305: The HI spectrum belongs to PGC $2308=$ IC 1562 (face on spiral at $V=3671 \mathrm{~km} \mathrm{~s}^{-1}$ ).

- PGC 31500: The HI spectrum belongs to PGC 31510 $\left(V=3799 \mathrm{~km} \mathrm{~s}^{-1}\right)$.
- PGC 51456: Possibly confused by PGC $51445=$ NGC $5595\left(V=2697 \mathrm{~km} \mathrm{~s}^{-1}\right) ; C_{\mathrm{p}}=0.15$ and $C_{\mathrm{v}}=$ 0.88 .

- PGC 70130: Confused by PGC $70127=$ MCG-1-589 and PGC $70133=$ MCG-1-58-11 $(V=3666$ and $3898 \mathrm{~km} \mathrm{~s}^{-1}$, respectively); $C_{\mathrm{p}}=0.77$ and $C_{\mathrm{v}}=0.99$.

Further, galaxies PGC 04353, PGC 42998, PGC 44589, PGC 05841, PGC 38712, PGC 44294, PGC 53965, PGC 28388, PGC 37719 may be confused by galaxies with unknown radial velocity and/or morphological type.

Acknowledgements. We express our gratitude to the observers of the Nançay radioastronomical observatory and also to N. Hallet and M. Loulergue for their helpful contribution. We would like to thank also Dr. W.K. Huchtmeier for his helpfull comments on the manuscript.

\section{References}

Bottinelli L., Gouguenheim L., Fouqué P., Paturel G., 1990, A\&AS 82, 391

de Vaucouleurs G., de Vaucouleurs A., Corwin H.G., 1976, Second Reference Catalogue of Bright Galaxies. University of Texas Press, Austin (RC2)

Fouqué P., Bottinelli L., Durand N., Gouguenheim L., Paturel G., 1990, A\&AS 86, 473

Paturel G., Fouqué P., Bottinelli L., Gouguenheim L., 1989a, Monographies de la base de données extragalactiques No. 1, Vols. I, II and III, ISBN 2.908288.00.1

Paturel G., 1981, ApJ 282, 382

Paturel G., Lanoix P., Teerikorpi P., et al., 1998, A\&A (in press)

Sandage A., 1996, AJ 111, 18

Teerikorpi P., 1975, A\&A 45, 117

Tully R.B., Fisher J.R., 1977, A\&A 54, 661 
Table 2. New HI data

SOSIE CANDIDATES OF NGC 224

\begin{tabular}{|c|c|c|c|c|c|c|c|c|c|c|c|}
\hline $\begin{array}{c}\text { PGC } \\
(1)\end{array}$ & $\begin{array}{l}\text { Name } \\
(2)\end{array}$ & $\begin{array}{l}\text { V20 } \\
(3)\end{array}$ & $\begin{array}{l}\text { V50 } \\
(4)\end{array}$ & $\begin{array}{c}\text { m.e. } \\
(5)\end{array}$ & $\begin{array}{c}\text { W20 } \\
(6)\end{array}$ & $\begin{array}{c}\text { m.e. } \\
(7)\end{array}$ & $\begin{array}{c}\text { W50 } \\
(8)\end{array}$ & $\begin{array}{c}\text { m.e. } \\
(9)\end{array}$ & $\begin{array}{c}\mathrm{FH} \\
(10)\end{array}$ & $\begin{array}{l}\text { m.e. } \\
\text { (11) }\end{array}$ & $\begin{array}{l}\mathrm{S} / \mathrm{N} \\
(12)\end{array}$ \\
\hline 01862 & M -2- 2-33 & 5363. & 5364 . & 7. & 320. & 21. & 309. & 14. & 2.9 & .8 & 4.6 \\
\hline 02358 & E $411-3$ & 6914. & 6924. & 7. & 425. & 21. & 395. & 14. & 4.3 & .6 & 8.0 \\
\hline 02791 & E $540-23$ & 6170. & 6170. & 7. & 448. & 20. & 432. & 13. & 7.4 & 1.2 & 6.1 \\
\hline 04353 & E 296- 6 & 6511. & 6505. & 10. & 346. & 30. & 328. & 20. & 4.3 & 1.1 & 4.3 \\
\hline 05581 & I 127 & 1873. & 1872. & 11. & 368. & 33. & 356. & 22. & 1.7 & .7 & 3.2 \\
\hline 05964 & E $353-26$ & 5402. & 5400. & 8. & 347. & 23. & 333. & 15. & 5.2 & 1.2 & 4.9 \\
\hline 06289 & M -3- 5-15 & 5515. & 5516. & 9. & 362. & 27. & 353. & 18. & 3.3 & 1.0 & 3.4 \\
\hline 07995 & M -1-6-43 & 5016. & 5018. & 8. & 387. & 24. & 374. & 16. & 3.4 & 1.0 & 4.6 \\
\hline 10595 & I 1853 & 4129. & 4132. & 5. & 421. & 15. & 392. & 10. & 15.5 & 1.4 & 10.7 \\
\hline 10829 & E $416-37$ & 5150. & 5150. & 11. & 453. & 33. & 437. & 22 . & 3.8 & 1.0 & 3.5 \\
\hline 11488 & N 1185 & 4912. & 4913. & 8. & 345. & 24. & 328. & 16. & 4.0 & .9 & 5.1 \\
\hline 18099 & E $488-44$ & 3339. & 3312. & 23. & 292. & 68. & 225. & 45. & 3.1 & 1.1 & 3.6 \\
\hline 35567 & E $439-17$ & 3799. & 3792. & 16. & 259. & 48. & 232. & 32. & 2.1 & .8 & 3.3 \\
\hline 37686 & N 4012 & 4182. & 4182. & 5. & 386. & 15. & 379. & 10. & 4.6 & .9 & 5.4 \\
\hline 42708 & N 4644 & 4900. & 4902. & 15. & 424. & 45. & 409. & 30. & 3.9 & 1.5 & 2.5 \\
\hline 42998 & N 4675 & 4793. & 4793. & 11. & 393. & 32. & 386. & 21. & 3.3 & 1.3 & 2.5 \\
\hline 44589 & E 507-62 & 3339. & 3339. & 8. & 330. & 23. & 317. & 15. & 5.5 & 1.2 & 4.6 \\
\hline 45241 & E $375-53$ & 2544 . & 2545 . & 2. & 235. & 7. & 226. & 5. & 6.5 & .7 & 12.1 \\
\hline 48393 & N 5267 & 6136. & 6148. & 21. & 192. & 63. & 164. & 42. & .7 & .4 & 2.5 \\
\hline 48908 & I 4325 & 3834. & 3834. & 7. & 342. & 21. & 320. & 14. & 1.5 & .3 & 6.9 \\
\hline 49993 & N 5443 & 1803. & 1808. & 8. & 371. & 23. & 361. & 16. & 5.2 & 1.3 & 4.1 \\
\hline 50182 & E $446-4$ & 4487. & 4489. & 8. & 231. & 23. & 221. & 16. & 4.0 & 1.3 & 4.1 \\
\hline 50987 & E 579-10 & 6419. & 6422. & 9. & 276. & 28. & 263. & 19. & 2.7 & .8 & 3.7 \\
\hline 54051 & E $448-13$ & 5692. & 5691. & 7. & 476. & 21. & 468. & 14. & 3.5 & .9 & 4.1 \\
\hline 67361 & M-1-55-11 & 5098. & 5088. & 8. & 332. & 23. & 300. & 15. & 5.7 & .8 & 7.5 \\
\hline
\end{tabular}

SOSIE CANDIDATES OF NGC 3031

\begin{tabular}{|c|c|c|c|c|c|c|c|c|c|c|c|}
\hline $\begin{array}{c}\text { PGC } \\
(1) \\
\end{array}$ & $\begin{array}{l}\text { Name } \\
(2) \\
\end{array}$ & $\begin{array}{c}\text { V20 } \\
(3) \\
\end{array}$ & $\begin{array}{l}\text { V50 } \\
(4) \\
\end{array}$ & $\begin{array}{c}\text { m.e. } \\
(5)\end{array}$ & $\begin{array}{c}\text { W20 } \\
(6) \\
\end{array}$ & $\begin{array}{c}\text { m.e. } \\
(7)\end{array}$ & $\begin{array}{c}\text { W50 } \\
(8) \\
\end{array}$ & $\begin{array}{c}\text { m.e. } \\
(9)\end{array}$ & $\begin{array}{l}\mathrm{FH} \\
(10) \\
\end{array}$ & $\begin{array}{l}\text { m.e. } \\
(11)\end{array}$ & $\begin{array}{l}\mathrm{S} / \mathrm{N} \\
(12) \\
\end{array}$ \\
\hline 00873 & $\mathrm{M}-1-1-49$ & 5417. & 5418. & 5. & 212. & 14. & 204. & 10. & 3.0 & .7 & 5.9 \\
\hline 02305 & I 1561 & 3764 . & 3764. & 2. & 54. & 6. & 35. & 4. & 4.2 & .5 & 22.1 \\
\hline 02642 & N 239 & 3743. & 3742. & 6. & 390. & 19. & 380. & 12. & 3.3 & .7 & 5.1 \\
\hline 03481 & M -3- 3-10 & 5558. & 5557. & 5. & 391. & 14. & 373. & 9. & 10.3 & 1.2 & 9.0 \\
\hline 11377 & E 546-37 & 4328. & 4339. & 12. & 206. & 37. & 171. & 24. & 1.8 & .6 & 4.8 \\
\hline 28388 & $\mathrm{M}-1-25-50$ & 6293. & 6299. & 14. & 380. & 42. & 357. & 28. & 2.7 & .9 & 3.4 \\
\hline 31500 & E $501-42$ & 3639. & 3649. & 8. & 116. & 25. & 77. & 17. & 2.0 & .5 & 7.5 \\
\hline 35608 & N 3752 & 1913. & 1913. & 12. & 233. & 36. & 212. & 24. & 3.1 & 1.1 & 3.8 \\
\hline 35954 & E $378-12$ & 2805. & 2799. & 9. & 304. & 27. & 285. & 18. & 5.9 & 1.3 & 4.8 \\
\hline 37719 & I 750 & 695. & 690 . & 7. & 378. & 20. & 350. & 13. & 18.6 & 2.2 & 7.9 \\
\hline 38582 & U 7129 & 947. & 947. & 10. & 160. & 30. & 152. & 20. & 1.1 & .7 & 2.7 \\
\hline 69734 & N 7378 & 2552. & 2560. & 11. & 263. & 33. & 235. & 22. & 1.8 & .5 & 4.9 \\
\hline
\end{tabular}


Table 2. continued

\begin{tabular}{|c|c|c|c|c|c|c|c|c|c|c|c|}
\hline $\begin{array}{c}\mathrm{PGC} \\
(1)\end{array}$ & $\begin{array}{l}\text { Name } \\
(2)\end{array}$ & $\begin{array}{l}\text { V20 } \\
(3)\end{array}$ & $\begin{array}{l}\text { V50 } \\
(4)\end{array}$ & $\begin{array}{c}\text { m.e. } \\
(5)\end{array}$ & $\begin{array}{c}\text { W20 } \\
(6)\end{array}$ & $\begin{array}{c}\text { m.e. } \\
(7)\end{array}$ & $\begin{array}{l}\text { W50 } \\
(8)\end{array}$ & $\begin{array}{c}\text { m.e. } \\
(9)\end{array}$ & $\begin{array}{c}\mathrm{FH} \\
(10)\end{array}$ & $\begin{array}{l}\text { m.e. } \\
\text { (11) }\end{array}$ & $\begin{array}{l}\mathrm{S} / \mathrm{N} \\
(12)\end{array}$ \\
\hline 05841 & E $476-25$ & 5802. & 5779. & 17. & 463. & 50. & 413. & 34. & 3.9 & .9 & 4.2 \\
\hline 06546 & N 671 & 5505 . & 5510 . & 13. & 418. & 40. & 390. & 27. & 2.5 & .7 & 4.0 \\
\hline 07743 & M -1- 6-31 & 4918. & 4913. & 9. & 294. & 26. & 272. & 17. & 3.8 & .9 & 5.3 \\
\hline 07917 & U 1572 & 3539 . & 3493. & 24. & 370. & 71. & 271. & 47. & 2.8 & .8 & 4.2 \\
\hline 08351 & U 1673 & 4494 . & 4474. & 10. & 462. & 29. & 421. & 19. & 3.2 & .5 & 6.6 \\
\hline 08480 & E $544-27$ & 2471. & 2460 . & 8. & 291. & 25. & 262. & 17. & 3.9 & .7 & 6.4 \\
\hline 08752 & I 1790 & 3611. & 3614. & 14. & 208. & 42. & 171. & 28. & 1.9 & .6 & 4.4 \\
\hline 11016 & E $356-18$ & 1438. & 1436. & 8. & 139. & 24. & 126. & 16. & 2.3 & .9 & 4.6 \\
\hline 13075 & E $482-1$ & 4307. & 4313. & 11. & 349. & 33. & 328. & 22. & 3.0 & .9 & 4.2 \\
\hline 15755 & U 3121 & 4489 . & 4495. & 12. & 476. & 37. & 459. & 24. & 3.0 & .8 & 3.4 \\
\hline 23708 & U 4412 & 6441. & 6444. & 9. & 366. & 26. & 356. & 17. & 2.2 & .7 & 3.5 \\
\hline 31919 & E $501-80$ & 1044. & 1048. & 3. & 171. & 8. & 134. & 5. & 17.8 & 1.2 & 23.3 \\
\hline 32703 & E $437-69$ & 3378. & 3378 . & 7. & 276. & 21. & 268. & 14. & 1.6 & .5 & 4.1 \\
\hline 33388 & U 6121 & 6455. & 6459. & 9. & 354. & 28. & 333. & 18. & 3.3 & .7 & 5.0 \\
\hline 33899 & E $377-17$ & 2433 . & 2436 . & 9. & 140. & 28. & 119. & 18. & 2.9 & .9 & 5.0 \\
\hline 35267 & E $439-11$ & 2345 . & 2331. & 12. & 260. & 37. & 182. & 25. & 4.5 & .8 & 7.1 \\
\hline 38126 & E 505- 9 & 2133. & 2133. & 8. & 154. & 24. & 140. & 16. & 2.7 & .8 & 4.8 \\
\hline 38712 & E $441-14$ & 2151. & 2171. & 10. & 272 . & 31. & 213. & 21. & 3.9 & .7 & 7.4 \\
\hline 42942 & N 4669 & 4833. & 4838. & 12. & 514. & 35. & 504. & 24. & 3.6 & 1.2 & 2.7 \\
\hline 44294 & E 507-50 & 4870 . & 4868. & 9. & 213. & 26. & 202. & 18. & 1.8 & .6 & 3.8 \\
\hline 46254 & I 4218 & 5804. & 5804. & 14. & 447. & 41. & 438. & 27. & 1.5 & .7 & 2.2 \\
\hline 46689 & E $444-14$ & 4155. & 4155. & 11. & 411. & 34. & 397. & 23. & 2.9 & .9 & 3.2 \\
\hline 48011 & N 5243 & 4160. & 4167. & 17. & 374. & 52. & 354. & 35. & 1.8 & .7 & 2.6 \\
\hline 49237 & U 8778 & 3221. & 3221. & 10. & 364. & 30. & 357. & 20. & 1.9 & .8 & 2.7 \\
\hline 49712 & N 5402 & 3044 . & 3046. & 12. & 285. & 36. & 265. & 24. & 3.2 & 1.1 & 3.7 \\
\hline 51305 & E $446-55$ & 2590 . & 2590. & 6. & 153. & 18. & 146. & 12. & 3.4 & 1.1 & 4.5 \\
\hline 52409 & N 5731 & 2546 . & 2546. & 6. & 272. & 19. & 221. & 13. & 10.5 & 1.1 & 11.3 \\
\hline 53562 & E 581-11 & 3255 . & 3258. & 6. & 262. & 17. & 248. & 11. & 6.3 & 1.2 & 6.6 \\
\hline 53861 & U 9703 & 2490 . & 2485. & 10. & 298. & 29. & 277. & 19. & 3.8 & .9 & 4.8 \\
\hline 53965 & N 5851 & 6508. & 6497. & 17. & 323. & 51. & 283. & 34. & 2.3 & .7 & 3.7 \\
\hline 58987 & E 586- 2 & 6614. & 6547. & 15. & 229. & 45. & 71. & 30. & 4.6 & 1.0 & 8.3 \\
\hline 61310 & U11097 & 4594. & 4584. & 10. & 356. & 30. & 309. & 20. & 4.7 & .8 & 6.8 \\
\hline 67813 & E $404-15$ & 4543. & 4543. & 7. & 347. & 22. & 341. & 15. & 2.0 & .7 & 3.3 \\
\hline 68360 & E $467-25$ & 4291. & 4293. & 10. & 234 . & 29. & 222. & 19. & 1.7 & .6 & 3.6 \\
\hline 71102 & U12519 & 4376. & 4372. & 16. & 329. & 48. & 304. & 32. & 3.7 & 1.2 & 3.1 \\
\hline
\end{tabular}

SOSIE CANDIDATES OF NGC 5457

\begin{tabular}{clcccccccccc}
\hline $\begin{array}{c}\text { PGC } \\
(1)\end{array}$ & $\begin{array}{l}\text { Name } \\
(2)\end{array}$ & $\begin{array}{c}\text { V20 } \\
(3)\end{array}$ & $\begin{array}{c}\text { V50 } \\
(4)\end{array}$ & $\begin{array}{c}\text { m.e. } \\
(5)\end{array}$ & $\begin{array}{c}\text { W20 } \\
(6)\end{array}$ & $\begin{array}{c}\text { m.e. } \\
(7)\end{array}$ & $\begin{array}{c}\text { W50 } \\
(8)\end{array}$ & $\begin{array}{c}\text { m.e. } \\
(9)\end{array}$ & $\begin{array}{c}\text { FH } \\
(10)\end{array}$ & $\begin{array}{c}\text { m.e. } \\
(11)\end{array}$ & $\begin{array}{c}\text { S/N } \\
(12)\end{array}$ \\
\hline 02269 & M -2- 2-73 & 5241. & 5242. & 6. & 204. & 17. & 172. & 11. & 4.5 & .7 & 10.0 \\
05321 & I 1702 & 4122. & 4132. & 8. & 277. & 24. & 241. & 16. & 2.7 & .5 & 7.5 \\
07942 & M-1- 6-41 & 5275. & 5265. & 8. & 154. & 23. & 126. & 15. & 2.1 & .5 & 6.8 \\
09315 & M -2- 7- 8 & 4944. & 4932. & 8. & 238. & 25. & 207. & 16. & 5.5 & 1.1 & 6.7 \\
16631 & U 3233 & 4661. & 4660. & 19. & 229. & 57. & 197. & 38. & .8 & .5 & 3.0 \\
26909 & I 539 & 7041. & 7042. & 8. & 258. & 23. & 247. & 15. & 2.3 & .7 & 4.4 \\
30657 & N 3249 & 3402. & 3397. & 8. & 181. & 25. & 171. & 17. & 2.4 & .8 & 3.8 \\
39623 & E 573-11 & 4006. & 4006. & 6. & 145. & 17. & 135. & 11. & 3.4 & 1.0 & 5.7 \\
51456 & N 5597 & 2669. & 2702. & 5. & 225. & 16. & 134. & 11. & 11.6 & .9 & 18.0 \\
70130 & M -1-58-10 & 3707. & 3684. & 3. & 237. & 10. & 95. & 7. & 15.1 & .7 & 35.1 \\
\hline
\end{tabular}

